\title{
CARACTERÍSTICAS CLÍNICAS, COMPORTAMENTAIS, COGNITIVAS E COMUNICATIVA NA SÍNDROME SMITH-MAGENIS
}

\author{
Clinical, cognitive, behavioral and communicative \\ features of Smith-Magenis Syndrome
}

\author{
Dionísia Aparecida Cusin Lamônica ${ }^{(1)}$, Greyce Kelly da Silva ${ }^{(2)}$, Renata Heloísa Furlan ${ }^{(3)}$, \\ Dagma Venturini Marques Abramides ${ }^{(4)}$, Gustavo Henrique Vieira ${ }^{(5)}$, \\ Danilo Moretti-Ferreira ${ }^{(6)}$, Célia Maria Giacheti ${ }^{(7)}$
}

\section{RESUMO}

Tema: o objetivo deste estudo foi descrever os aspectos clínico, comportamental, cognitivo e comunicativo de indivíduos com o diagnóstico genético da Síndrome Smith-Magenis. Procedimentos: participaram dois indivíduos do sexo masculino, de nove e 19 anos. Realizou-se a avaliação genética clínica e laboratorial (teste FISH, utilizando sonda para região 17p11.2). A avaliação psicológica constou da observação comportamental e aplicação da Escala Wechsler de Inteligência. A avaliação Fonoaudiológica foi realizada por meio de procedimentos formais e informais e avaliação auditiva periférica. Resultados: a análise genética clínica evidenciou as características fenotípicas da síndrome Smith-Magenis, confirmada pela avaliação laboratorial. A avaliação psicológica evidenciou o fenótipo comportamental peculiar da síndrome Smith-Magenis e comprovou a deficiência intelectual de grau moderado nos dois indivíduos. A avaliação fonoaudiológica mostrou alterações no desempenho linguístico, com alterações nos níveis fonológico, semântico, sintático e pragmático e nas habilidades psicolinguísticas, interferindo nas habilidades comunicativas e de aprendizagem. A avaliação auditiva indicou audição periférica dentro de parâmetros de normalidade. Conclusão: a avaliação multidisciplinar favoreceu a descrição dos aspectos clínicos, comportamentais, cognitivos que pertencem ao fenótipo comportamental da síndrome Smith-Magenis e permitiu verificar que estes apresentam graves alterações da linguagem oral, das habilidades psicolinguísticas e do processamento das informações visuais e auditivas com reflexos marcantes no desenvolvimento das habilidades comunicativas e processos de aprendizagem.

DESCRITORES: Linguagem; Cognição; Genética; Comportamento

(1) Fonoaudióloga; Professora Associada do Departamento de Fonoaudiologia da Faculdade de Odontologia de Bauru da Universidade de São Paulo - FOB/USP, Bauru, São Paulo, Brasil; Livre Docente.

(2) Fonoaudióloga, Bauru, São Paulo, Brasil; Mestre em Fonoaudiologia pela Faculdade de Odontologia de Bauru da Universidade de São Paulo.

(3) Fonoaudióloga do Hospital de Reabilitação de Anomalias Craniofaciais da Universidade de São Paulo, Bauru, São Paulo, Brasil; Mestranda em Ciências da Reabilitação pelo Hospital de Reabilitação de Anomalias Craniofaciais da Universidade de São Paulo.

(4) Psicóloga; Professora Doutora do Departamento de Fonoaudiologia da Faculdade de Odontologia de Bauru da Universidade de São Paulo - FOB/USP.

(5) Biomédico; Doutorando do Programa de Pós-graduação em Ciências Biológicas- Área de Concentração: Genética da Universidade Estadual Paulista -

\section{INTRODUÇÃO}

A Síndrome Smith-Magenis (SSM) é uma afecção genética caracterizada por múltiplas anomalias congênitas e alterações comportamentais,

UNESP - Botucatu, Brasil; Mestre em Ciências Biológicas (Genética) pela Instituto de Biociências da Universidade Estadual Paulista-UNESP-Botucatu-SP.

(6) Biomédico; Professor Adjunto do Departamento de Genética do Instituto de Biociências de Botucatu, UNESP.da Universidade Estadual Paulista - UNESP-Botucatu, São Paulo, Brasil; Livre Docente.

(7) Fonoaudióloga, Professor Adjunto do Departamento de Fonoaudiologia da Universidade Estadual Paulista UNESP-Marília; São Paulo, Brasil; Livre Docente.

Conflito de interesses: inexistente 
associadas à deleção da região cromossômica $17 p 11.2$ ou mutações do ponto gene $R A / 1^{1-9}$.

Os dismorfismos craniofaciais englobam braquicefalia, fronte alargada, hipoplasia da linha média da face, lábio superior proeminente com aspecto de arco, fenda palpebral desviada para cima, distância dos olhos diminuída, face alargada e quadrada, prognatismo, sobrancelhas espessas e sinofre. Outras anomalias observadas são braquidactilia, baixa estatura, pés planos, escoliose, pele áspera ${ }^{2,3,6-10}$, atraso global do desenvolvimento, deficiência intelectual moderada, alterações eletroencefalográficas, hipotonia na infância, alterações visuais, perda da audição, anomalias cardíacas, renais e esqueléticas e distúrbios do sono 4-6,10-15.

As manifestações comportamentais incluem: hiperatividade, impulsividade, dificuldades em comportamentos adaptativos, déficit de atenção, auto-estimulação, auto-agressão, com acessos de raiva, mau humor e dificuldade com asseio corporal, comportamentos de "auto-abraço" e lamber as mãos, enquanto folheia páginas. Apresentam interesses restritos, tendência a isolamento, e comportamentos autísticos com dificuldades quanto a mudanças de rotina $a^{5,6,9,12-14}$. Outros comportamentos comuns referem-se à poliembolocoilamania, e onicotilomania ${ }^{5,8,9,15-17}$.

Estes indivíduos apresentam deficiência intelectual moderada, com média de inteligência entre 40 a 50 pontos ${ }^{6,10,11-13}$. Quanto às habilidades de comunicação, muitos pacientes parecem ter menos dificuldades nas habilidades receptivas do que expressivas ${ }^{4-6,12-14}$. O atraso no desenvolvimento da linguagem e fala é evidente ${ }^{13}$. A descrição quanto aos níveis linguísticos, na revisão da literatura, é vaga, não existindo trabalhos com este enfoque.

No quadro clínico da SSM também são observadas alterações visuais (opacificação corneal, catarata, miopia, estrabismo) e auditivas. A literatura apresenta que cerca de $68 \%$ dos indivíduos com a SSM apresentam perda auditiva ${ }^{4-6,13,14}$. Infecções da orelha média são frequentes, levando a perda auditiva condutiva $a^{3,6,9,11,18,19}$. É possível a ocorrência de perda auditiva neurossensorial progressiva com sinais de neuropatias periféricas e diminuição de reflexos ${ }^{11,18,19}$.

Considerando o exposto, o objetivo deste estudo foi descrever os aspectos clínicos, comportamentais, cognitivo e comunicativo de dois indivíduos com o diagnóstico genético da Síndrome Smith-Magenis, visando estabelecer a correlação fenótipo-genótipo.

\section{APRESENTAÇÃO DO CASO}

Este estudo foi aprovado pelo Comitê de Ética em Pesquisa com Seres Humanos da Instituição onde o estudo foi realizado no Serviço de Aconselhamento Genético-Instituto de Biociências de Botucatu/Universidade Estadual Paulista "Júlio de Mesquita Filho" (SAG-IBB/UNESP) (protocolo número 447/2007). As famílias dos participantes assinaram o Termo de Consentimento Livre e Esclarecido. Cumpriram-se todos os quesitos propostos na Resolução 196/96 que versa sobre estudos com seres humanos.

\section{Casuística}

Este estudo teve a participação de dois indivíduos encaminhados ao Serviço de Aconselhamento Genético do IBB/UNESP-Botucatu para avaliação clínica e citogenética. Ambos do sexo masculino, não aparentados, com 9 (nove) anos de idade - S1; e 19 (dezenove) anos de idade - S2.

As informações do histórico do desenvolvimento foram fornecidas pelos responsáveis legais dos participantes:

S1: Filho único de casal jovem não consanguíneo. Avaliado aos nove anos. Seus antecedentes peri-natais não revelaram anormalidades. Nasceu de parto cesariana, com $3.050 \mathrm{gr}, 47 \mathrm{~cm}$ e perímetro cefálico de $36 \mathrm{~cm}$. Apresentou dificuldades alimentares, refluxo gastresofágico, otites recorrentes e atraso no desenvolvimento neuropsicomotor e da linguagem (sentou aos 9 meses, andou aos 15 meses, primeiras palavras após os 24 meses e dificuldade para formar frases até a presente idade). Negou crises convulsivas. É agitado, hiperativo, impulsivo e apresenta distúrbios do sono. Foram relatados problemas comportamentais peculiares. Gosta de desmontar aparelhos eletrônicos. Apresenta movimentos estereotipados de abraçar-se, auto-estimulação de órgão genital, poliembolocoilamania, e onicotilomania. Fez uso de diversos medicamentos com melhora temporária do comportamento. A avaliação oftalmológica indicou distúrbio refrativo e opacificação corneal periférica, entretanto, há dificuldade para utilização de lentes corretivas, devido aos transtornos de comportamento. Foi transferido de várias escolas por dificuldades de adaptação escolar, problemas de comportamento e de aprendizagem. Atualmente frequenta escola especial.

S2: Filho da segunda união da mãe. Avaliado aos 19 anos. Mãe relatou aborto espontâneo anterior a esta gestação. Fez uso de medicamento orientado pelo médico, pois o feto teve diminuição 
de movimentos durante o $4^{\circ}$ mês gestacional. Nasceu de parto cesariana, a termo, com 2.500gr e $42 \mathrm{~cm}$. Apresentou atraso no desenvolvimento neuropsicomotor e de linguagem, com marcha por volta dos 27 meses e as primeiras palavras após os 36 meses. Apresentou dificuldades de alimentação, refluxo gastresofágico e distúrbio do sono. Sempre apresentou fala ininteligível e com velocidade acelerada. Demonstrava dificuldades com mudança de rotina, comportamento agressivo, comportamento de lamber as mãos enquanto folheia páginas, onicotilomania e poliembolocoilamania. A avaliação oftalmológica, realizada por volta dos cinco anos, indicou miopia. Faz uso de lentes corretivas desde esta data. Aos seis anos apresentou convulsões, com controle medicamentoso. Apresentou otites recorrentes. Em relação à aprendizagem, estudou em escola regular até a $3^{\mathrm{a}}$ série sem aproveitamento. Há queixas de dificuldade de adaptação escolar e problemas de comportamento. Atualmente frequenta escola especial.
Os dois participantes foram submetidos às seguintes avaliações:

\section{Avaliação Genética-Clínica}

Foi realizada anamnese genético-clinica padrão, com ênfase em histórico obstétrico, perinatal, pediátrico, familial, propedêutica especializada e obtenção de dados antropométricos.

\section{Avaliação Citogenética}

Foram realizados cariótipos de sangue periférico dos dois pacientes. A análise citogenética por bandamento GTG (400 bandas) revelaram cariótipos 46,XY, del(17)(p11.2p11.2)- Figura 1(a e b). Em ambos os casos foram realizados exames de citogenética molecular por Hibridação in situ Fluorescente (FISH), utilizando sondas comeciais da Cytocell ${ }^{\circledR}$ para detecção dos genes RAl1 e FLII, cujos cariótipos foram 46,XY. Fish, del(17) (P11.2P11.2)(RAl1-;FLII-)

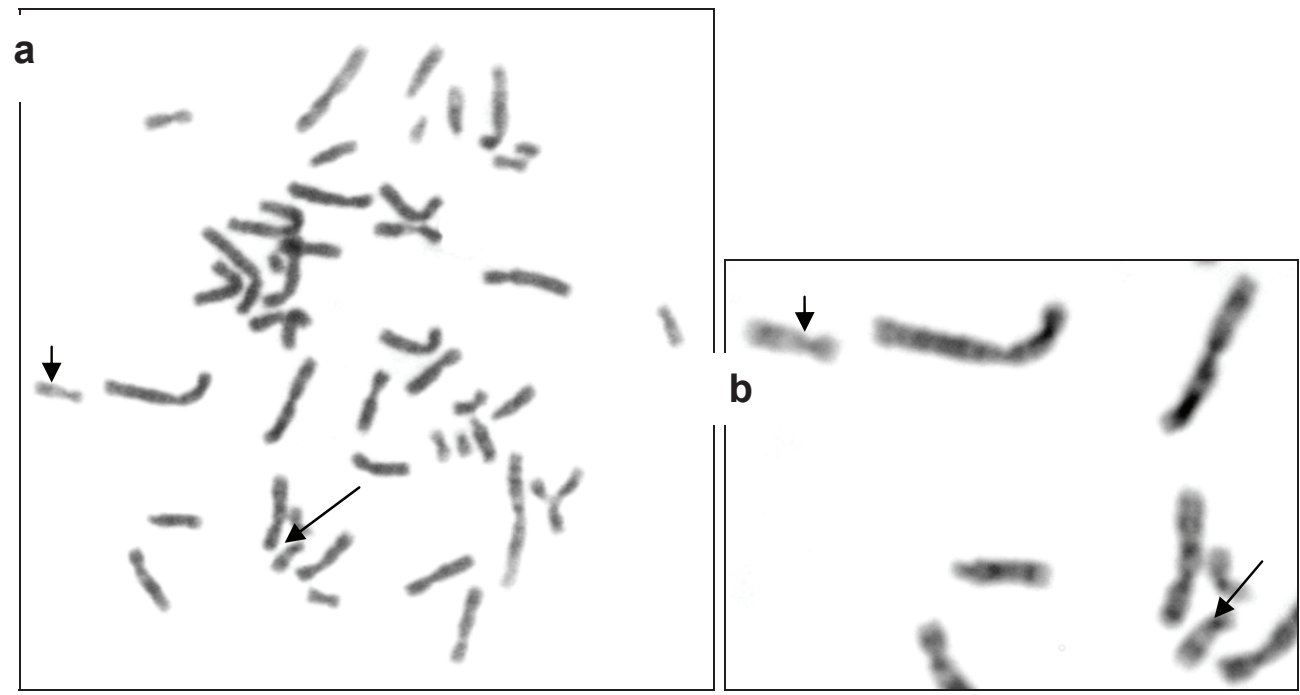

(a) metáfase em bandamento GTG com resolução aproximada de 400 bandas do caso S1

(b) Ilustração parcial da mesma metáfase indicando os cromossomos 17

Nota: A seta menor indica o cromossomo 17 normal e a seta maior indica o cromossomo 17 deletado

Figura 1 (a e b) - Resultado citogenético bandamento GTG

\section{Avaliação psicológica}

Foi realizada a observação comportamental e aplicada a Escala Wechsler de Inteligência para Crianças $^{20}$ (WISC-III) e para Adultos (WAIS) ${ }^{21}$.

\section{Avaliação Fonoaudiológica}

Foi realizada observação do comportamento comunicativo, aplicado o Teste Illinois de
Habilidades Psicolinguística (ITPA) ${ }^{22}$, o Teste de Vocabulário por Imagem Peabody (TVIP) ${ }^{23}$, o Teste de Linguagem Infantil: Nas Áreas de Fonologia, Vocabulário, Fluência e Pragmática (ABFW) - área de fonologia ${ }^{24}$. Também foi realizada a avaliação audiológica por meio de audiometria tonal liminar ${ }^{25} \mathrm{e}$ da medida da imitância acústica. Os equipamentos utilizados para a avaliação audiológica foram: SD 50, marca Siemens e SD 30, marca Siemens. 


\section{RESULTADOS}

A Tabela 1 apresenta os principais sinais clínicos observados nos participantes com a síndrome de Smith-Magenis da presente casuística e a frequência destes sinais descritos na literatura.

\section{Avaliação psicológica}

S1: Na avaliação psicológica foi observado alteração nos processos atencionais e comportamento desatento. Encontra-se em fase intermediária entre o concreto e o simbólico, não consolidando a função representativa. O resultado na Escala Wechsler de Inteligência ${ }^{20}$ evidenciou escores compatíveis com deficiência intelectual moderada $(\mathrm{Ql}=47)$. Na observação do comportamento comunicativo observouse hiperatividade, impulsividade, comportamentos mal adaptativos, auto-estimulação, momentos de agressividade, interesses restritos, poliembolocoilamania, onicofagia, onicotilomania e deficiência intelectual.

S2: Alteração nos processos atencionais e comportamento desatento. Apresentou insegurança frente às situações novas e frustração frente às dificuldades para realizar atividades propostas. $O$ resultado na Escala Wechsler de Inteligência ${ }^{21}$ evidenciou escores compatíveis com deficiência intelectual moderada $(\mathrm{QI}=44)$. Na observação do comportamento comunicativo observou-se hiperatividade, impulsividade, comportamentos mal adaptativos, auto-estimulação, momentos de agressividade, tendência ao isolamento, interesses restritos, poliembolocoilamania, onicofagia, onicotilomania e deficiência intelectual.

Tabela 1 - Principais sinais clínicos da síndrome de Smith-Magenis verificados no presente trabalho e frequência de ocorrência descrita na literatura (\%) *

\begin{tabular}{|c|c|c|c|}
\hline Sinais Clínicos & S1 & S2 & $\%$ \\
\hline \multicolumn{4}{|l|}{ Craniofacial/músculo esquelético } \\
\hline Braquicefalia & + & + & 90 \\
\hline Hipoplasia de face média & + & + & 90 \\
\hline Prognatismo relativo com a idade & - & + & 50 \\
\hline Face larga e de formato quadrado & + & + & 80 \\
\hline Sinofre & + & + & $30-65$ \\
\hline Lábio superior protuso e em " $v$ " invertido & + & + & $70-90$ \\
\hline Baixa estatura & + & + & 70 \\
\hline Escoliose & - & + & $40-70$ \\
\hline \multicolumn{4}{|l|}{ Otorrinolaringológica } \\
\hline Anomalias de laringe e orelha média & - & - & $80-90$ \\
\hline Voz rouca e grave & + & + & 80 \\
\hline \multicolumn{4}{|l|}{ Neuro-comportamental } \\
\hline Comprometimento cognitivo/ & + & + & 100 \\
\hline Atraso no desenvolvimento & + & + & 100 \\
\hline Hipotonia (até os 2 anos) & + & + & 90 \\
\hline Distúrbio do sono & + & + & 90 \\
\hline Atraso de fala & + & + & 90 \\
\hline Agressividade & + & + & $80-100$ \\
\hline Sinal de auto-agressão & + & + & $70-90$ \\
\hline Lamber os dedos como se folheasse páginas & + & $50-80$ & \\
\hline Auto-abraço & + & + & $50-80$ \\
\hline Poliembolocoilomania & + & + & $25-85$ \\
\hline Onicotilomania & + & + & $25-85$ \\
\hline \multicolumn{4}{|l|}{ Aspectos comuns } \\
\hline Obesidade & + & - & 13 \\
\hline Anormalidades odontológicas & + & 90 & \\
\hline
\end{tabular}

*Nota: modificada ${ }^{(16)}$ 


\section{Avaliação Fonoaudiológica:}

Observação do comportamento comunicativo:

S1: Compreende ordens simples em contextos imediatos e concretos e se expressa por meio de palavras isoladas seguidas de gestos indicativos. Apresenta dificuldade no controle do comportamento. É impulsivo e hiperativo. Apresenta movimentos estereotipados (abraçar-se, autoestimulação de genital, poliembolocoilamania e onicofagia). A falta de atenção dificultou a realização das atividades. Manipula objetos de forma superficial e rápida. Apresentou dificuldade no reconhecimento de letras.

S2: Compreende ordens simples em contextos imediatos e concretos e responde com palavras isoladas ou frases simples. Inicia turnos, responde e mantém atividade dialógica relacionada a temas da vida diária, embora tenha dificuldade para manutenção de tópicos conversacionais. A realização de atividades é dificultada pelos comportamentos maladaptativos (impulsividade, agressividade, flapping, onicotilomania, onicofagia e poliembolocoilamania). Apresentou dificuldades para realizar atividades ligadas à leitura e a escrita.

A Tabela 2 apresenta os escores obtidos quanto a Idade Psicolinguística em cada um dos subtestes do ITPA.

Na aplicação do TVIP S1 apresentou pontuação baixa inferior e $\mathbf{S} 2$ baixa superior para a habilidade de recepção auditiva.

$\mathrm{Na}$ avaliação fonológica foi observado que S1 apresentou redução da palavra, à sílaba tônica, frontalização de velares, simplificação de encontro consonantal e consoante final. $\mathrm{Na}$ prova de imitação não houve melhora dos padrões fonológicos. S2 apresentou posteriorização para palatal, simplificação de encontro consonantal e consoante final. Na prova de imitação não houve melhora dos padrões fonológicos.

A avaliação audiológica periférica indicou resultados compatíveis com normalidade tanto para S1 quanto para S2.

\section{DISCUSSÃO}

A SSM foi descrita em $1982^{1}$ e sua incidência é estimada em 1:25.000 nascidos vivos, entretanto, estudos relatam que esta síndrome ainda é pouco diagnosticada pelo desconhecimento dos profissionais de saúde ${ }^{6,12}$.

Neste estudo, os participantes apresentaram características clínicas peculiares da SSM e os exames genéticos laboratoriais confirmaram o diagnóstico ${ }^{1-19}$.

A avaliação psicológica confirmou a deficiência intelectual descrita na SSM ${ }^{12-14,17}$, de grau moderado e os também os transtornos comportamentais, como a hiperatividade, a impulsividade, os comportamentos de isolamento, a agressividade, os acessos de raiva, o transtorno do humor e as dificuldades com mudanças de rotina. Também foram observados os comportamentos de poliembolocoilamania, onicotilomania e onicofagia ${ }^{6-9,12-15}$. A literatura apresenta que o fenótipo comportamental ${ }^{3-5,10,11-17}$, evidente a partir da idade escolar, representa um grande problema no cuidado diário com estes indivíduos, tanto para pais quanto para professores ${ }^{5,18}$, dificultando a inserção destas

Tabela 2 - Idade psicolinguística nos subtestes do teste de Illinois de habilidades psicolinguística (ITPA)

\begin{tabular}{lcc}
\hline Subtestes & S1 & S2 \\
\hline Recepção Auditiva & 3 anos 9 meses & 7 anos e 3 meses \\
Recepção Visual & 4 anos 9 meses & 5 anos \\
Associação Auditiva & 3 anos 4 meses & 6 anos \\
Associação Visual & 4 anos e 4 meses & 7 anos e 6 meses \\
Expressão Verbal & Não obteve base & Não obteve base \\
Expressão Manual & 4 anos & 4 anos \\
Memória Auditiva & Não obteve base & 3 anos e 6 meses \\
Memória Visual & Não obteve base & Não obteve base \\
Closura Gramatical & Não obteve base & 4 anos \\
Closura Visual & 6 anos & 7 anos e 6 meses \\
Closura Auditiva & Não obteve base & 6 anos e 4 meses \\
Combinação de sons & Não obteve base & 2 anos \\
\hline
\end{tabular}


crianças nos diferentes ambientes sociais e nas interações interpessoais ${ }^{7,8,13,17}$, trazendo reflexos para as habilidades comunicativas e socialização ${ }^{12}$.

Alguns indivíduos com SSM, podem preencher critérios para diagnóstico de autismo ${ }^{3,7,9,15,16}$, como por exemplo, resistência a mudanças de rotina, estereotipias motoras, comportamentos mal adaptativos, repetitivos e de isolamento. Estes comportamentos foram observados nos participantes deste estudo. Entretanto, um estudo ${ }^{12}$ desta temática revelou a necessidade do diagnóstico correto, pois há diferenças cruciais entre estas entidades diagnósticas.

É necessário atenção ao fenótipo de indivíduos com a SSM, principalmente relacionado às características comportamentais, para confirmação do diagnóstico, por meio da avaliação citogenética molecular, a fim de possibilitar o diagnóstico correto e providenciar medidas interventivas que favoreçam o desenvolvimento destes indivíduos ${ }^{2-4,9,19}$.

$\mathrm{Na}$ observação do comportamento comunicativo observou-se que a linguagem está alterada nos níveis fonológico, semântico, sintático e pragmático para S1 e S2. Também foram observadas alterações nas habilidades psicolinguísticas (Tabela 2), ou seja, S1 apresentou escores abaixo do esperado para sua idade cronológica nos subtestes de recepção auditiva e visual, associação auditiva e visual, expressão manual e closura visual, não obtendo base nos seguintes subtestes: expressão verbal, memória auditiva e visual, closura gramatical, closura auditiva e combinação de sons. S2 também apresentou escores abaixo do esperado para sua idade cronológica em todos os subtestes e não conseguiu base nos subtestes de expressão verbal e memória visual. Alterações psicolinguísticas interferem no processamento das informações que somadas às alterações comunicativas $\mathrm{e}$ déficits cognitivos trazem reflexos negativos para o desenvolvimento de habilidades comunicativas e de aprendizagem.

O desempenho dos participantes na prova de vocabulário receptivo foi aquém do esperado. $\mathrm{Na}$ recepção oral observou-se adequação somente quando utilizado conteúdos concretos e imediatos. S1 e S2 apresentaram dificuldades para compreender conteúdos relacionados a eventos não presenciais, figuras de linguagem e anedotas. Quanto às habilidades expressivas, S1 fala apenas palavras isoladas, com processos fonológicos não esperados para sua faixa etária e S2, apesar de formar frases simples, relatando contextos imediatos e concretos, apresenta dificuldade para manter o tema conversacional e troca de turnos, também apresentando processos fonológicos não esperados para sua faixa etária.
Quanto às habilidades de linguagem escrita, S1 apresenta reconhecimento de letras e S2 consegue escrever palavras simples, demonstrando que estas habilidades estão aquém, considerando a idade cronológica e principalmente o tempo de escolaridade. A literatura é escassa quanto ao detalhamento das habilidades de linguagem tanto falada quanto escrita, principalmente em estudos que apresentam detalhamento dos níveis linguísticos. Um estudo ${ }^{6}$ relatou que em indivíduos com SSM o atraso no desenvolvimento da fala, com ou sem perda auditiva, ocorre em aproximadamente 96\% dos casos e que as habilidades de linguagem receptivas parecem ser melhores do que às expressivas. Atraso de fala e disfunção motora oral foram relatados em outros estudos ${ }^{4-6}$.

Quanto à audição periférica, tanto S1 quanto S2 apresentaram resultados compatíveis com normalidade. O desempenho nas habilidades auditivas e visuais encontrou-se bastante prejudicado para os dois participantes. Segundo a literatura compilada é esperado, na SSM, dificuldades na integração sensorial ${ }^{4,11,12}$, o que poderia explicar as alterações encontradas. Outro estudo ${ }^{11}$ relatou que os profissionais devem estar alertas para os sintomas de progressão ou flutuação das habilidades auditivas. Ressalta-se que muitas vezes a flutuação das habilidades auditivas está relacionada à diminuição do tempo de atenção e interesses restritos às atividades interativas e dialógicas. O acompanhamento longitudinal nestes casos é extremamente necessário, uma vez que podem ocorrer, como apresentado na literatura, perda auditiva neurossensorial progressiva com sinais de neuropatia periférica e diminuição de reflexos ${ }^{11,19}$.

Os prejuízos na visão e audição e no processamento das informações ${ }^{4,19}$, além de outros fatores, afetam as habilidades de comunicação. Estes outros fatores referem-se aos comportamentos mal adaptativos, ao déficit atencional e a deficiência intelectual.

Vários estudos apresentaram que o perfil cognitivo da SSM caracteriza-se por alteração na modulação das respostas do imput sensorial, debilidade nos processos atencionais, dificuldade em atividades que exigem atenção sustentada, no processamento sequencial e na memória de curto prazo. Os achados deste estudo corroboram com os encontrados na literatura ${ }^{5,6,11}$. O quadro clínico da síndrome Smith-Magenis é complexo e envolve aspectos multifatoriais, com manifestações comportamentais, cognitivas e comunicativas graves, interferindo no processamento das informações, na aprendizagem de conteúdo e na integração social e educacional destes indivíduos. 
Ressalta-se a importância do conhecimento desta entidade clínica para o diagnóstico adequado e a realização de estratégias terapêuticas que favoreçam o desenvolvimento destes indivíduos.

\section{CONCLUSÃO}

As características clínicas encontradas no S1 e S2 foram: alterações esqueléticas, dismorfismo craniofaciais, braquicefalia, hipoplasia da linha média da face, fronte proeminente, lábio superior com aspecto de arco, fenda palpebral desviada para cima, distância dos olhos diminuída, face alargada e quadrada, prognatismo, sobrancelhas espessas e sinofres evidenciando características peculiares da SSM. Quanto às alterações comportamentais foram observados comportamentos mal adaptativos, hiperatividade, impulsividade, agressividade, autoestimulação, poliembolocoilamania, onicotilomania e onicofagia. Ambos apresentaram deficiência intelectual moderada. Tanto S1 quanto S2 apresentaram dificuldades importantes nas habilidades receptivas e expressivas da linguagem, com alterações semânticas, fonológicas, sintáticas e pragmáticas. Na avaliação audiológica os resultados encontram-se dentro dos padrões de normalidade. Alterações no processamento das informações visuais e auditivas, também foram encontradas.

\begin{abstract}
Background: this study aimed to describe the clinical, behavioral, cognitive and communicative features of subjects with Smith-Magenis Syndrome genetic diagnosis. Procedures: the subjects were two males, 09 and 19 year old. We performed a clinical and laboratory genetic evaluation (FISH assay using probes for the region 17p11.2). The psychological evaluation consisted of behavioral observation and application of the Wechsler Intelligence Scale. Speech evaluation was performed by means of formal and informal procedures and peripheral hearing evaluation. Results: the clinical genetic analysis showed the phenotypic characteristics of Smith-Magenis syndrome, confirmed by laboratory evaluation. The psychological evaluation revealed the peculiar phenotype behavioral of Smith-Magenis syndrome and confirmed the moderate intellectual disabilities in two subjects. Speech evaluation showed changes in language performance, with changes in phonological, semantic, syntactic and pragmatic levels and psycholinguistic skills, interfering with communication and learning skills. The hearing test showed peripheral hearing within normal parameters. Conclusion: the multidisciplinary approach made easier the description of clinical, behavioral, cognitive aspects, belonging to the behavioral phenotype of Smith-Magenis syndrome and showed that these changes have severe oral language alterations in skills and psycholinguistic processing of visual and auditory information with remarkable consequences on the development of communicative skills and learning processes.
\end{abstract}

KEYWORDS: Language; Cognition; Genetics; Behavior

\section{REFERÊNCIAS}

1. Smith ACM, McGavran L, Waldstein G. Deletion of the 17 short arm in two patients with facial clefts. Am J Hum Genet. 1982; 34:410A.

2. Vlangos $\mathrm{CN}$, Wilson $\mathrm{M}$, Blancato $\mathrm{J}$, Smith $\mathrm{ACM}$, Elsea SH. Diagnostic FISH probes for del 17(p11.2p.11.2) associated with Smith-Magenis syndrome should contin the RAl1 gene. Am J Med Genet A. 2005;132A(3):278-82.

3. Taylor L, Oliver C. The behavioral phenotype of Smith-Magenis syndrome: evidence for a geneenvironment interaction. J Intellect Disabil Res. 2008;52(10):830-41.
4. Edelman EA, Girirajan S, Finucane, B, Patel PI, Lupsky JR, Smith ACM, et al. Gender, genotype, and phenotype differences in Smith-Magesis syndrome: a meta-analysis of 105 cases. Clin Genet. 2007;71(6): 540-50.

5. Bronberg R, Ziembar M, Drut M, Goldschmidt E. Smith-Magenis syndrome: comunicacíon de un caso y revision de la bibliografía. Arch Argent Pediatr. 2008;16(2)143-54.

6. Elsea SH, Girirajan S. Smith-Magenis syndrome. Eur J Hum Genet. 2008;16(4):412-21.

7. Taylor L, Oliver C. The behavioral phenotype of Smith-Magenis syndrome: evidence for a gene- 
environment interaction. J Intellect Disabil Res. 2008;52(10)830-41.

8. Boudreau EA, Johnson KP, Jackman AR, Blancato J, Huizing M, Bendavid C, et al. Review of disrupted sleep patterns in Smith-Magenis syndrome and normal melatonin secretion in a patient with an atypical interstitial 17p11.2 deletion. Am J Med Genet A. 2009;149A(7):1382-91.

9. Williams SR, Girirajan S, Tegay D, Nowak $\mathrm{N}$, Hatchwell E, Elsea SH. Array comparative genomic hybridisation of 52 subjects with a SmithMagenis-like phenotype: identification of dosage sensitive loci also associated with schizophrenia, autism, and developmental delay. J Med Genet. 2010;47(4):223-9.

10. Gropman AL, Duncan WC, Smith AC. Neurologic and developmental features of the Smith-Magenis syndrome (del 17p11.2). Pediatr Neurol. 2006;34(5):337-50.

11. Di Cicco M, Padoan R, Felisati G, Dilani D, Moretti E, Guerneri S, et al. Otorhinolaringologic manifestations of Smith-Magenis syndrome. Int J Pediatr Otorhinolaryngol. 2001;59(2):147-50.

12. Gropman AL, Duncan WC, Smith ACM. Neurological and developmental features of the Smith-Magenis syndrome (del 17p11.2).Pediatric Neurol. 2006;34(5):337-50.

13. Wolters PL, Gropman AL, Martin SC, Smith MR, Hildenbrand HL, Brewer CC, et al. Neurodevelopment of children under 3 years of age with Smith-Magenis syndrome.Pediatr Neurol. 2009;41(4):250-8.

14. Gropman AL, Elsea S, Duncan Junior WC, Smith AC. New developments in Smith-Magenis syndrome (del 17p11.2). Curr Opin Neurol. 2007;20(2):125-34. 15. Martin SC, Wolters PL, Smith ACM. Adaptative and maladaptative behavior in children with Smith-Magenis syndrome. J Autism Dev Disord. 2006;36(4):541-52.
16. Foster RH, Kozachek S, Stern M, Elsea SH. Caring for the caregivers: an investigation of factors related to well-being among parents caring for a child with Smith-Magenis syndrome. J Genet Couns. 2010;19(2):187-98.

17. Madduri N, Peters SU, Voigt RG, Llorent AM, Lupski JR, Potocki, L. Cognitive and adaptive behavior profiles in Smith-Magenis syndrome. $J$ Dev Behav Pediatr. 2006;27(3):188-92.

18. Greenberg G, Lewis RA, Potocki L, Glaze D, Parke J, Killian J, et al. Multi-disciplinary clinical study of Smith-Magenis syndrome (deletion 17p11.2). Am J Med Genet. 1996;62(3):247-54.

19. Liburd N, Ghosh M, Riazuddin S, Naz S, Khan S, Ahmed Z, et al. Novel mutations of MYO15A associated with profound deafness in consanguineous families and moderately severe hearing loss in a patient with Smith-Magenis syndrome. Hum Genet. 2001;109(5):535-41.

20. Wechsler D. WISC - escala de inteligência Wechsler para crianças: manual de aplicação. Rio de Janeiro: CEPA; s.d.

21. Wechsler D. Wechsler abbreviated scale of intelligence manual. San Antonio: Psychological Corporation; 1999.

22. Bogossian MADS, Santos MJ. Manual do examinador: teste Illinois de habilidades psicolingüísticas. Rio de Janeiro: EMPSI; 1977.

23. Dunn LM, Padilla ER, Lugo DE, Dunn LM. Test de vocabulario en imagens Peabody: adaptación hispanoamericana. Circle Pines: Dunn Educational Services; 1986.

24. Wertzner HF. Fonologia. In: Andrade CR, BefiLopes DM, Fernandes FDM, Wertzner HF. ABFW: teste de linguagem infantil nas áreas de fonologia, vocabulário, fluência e pragmática. 2a. ed. Barueri: Pró-Fono; 2004. p.5-32.

25. Daves H, Silverman SR. Hearing and deafness. New York: Holt; 1970.

http://dx.doi.org/10.1590/S1516-18462011005000118

RECEBIDO EM: 14/12/2010

ACEITO EM: 27/05/2011

Endereço para correspondência:

Dionísia Aparecida Cusin Lamônica

Departamento de Fonoaudiologia.

Alameda Octávio Pinheiro Brisolla 9-75

Bauru, SP, Brasil

CEP: 17012-901

E-mail: dionelam@uol.com.br 